\title{
Ginseng-Angelica-Sansheng-Pulvis Boosts Neurogenesis Against Focal Cerebral Ischemia-Induced Neurological Deficiency
}

\author{
Bowen Liu' ${ }^{1}$, Qian Zhang ${ }^{2}$, Chienchih Ke ${ }^{3,4}$, Zhenyan Xia ${ }^{5}$, Cheng Luo ${ }^{5}$, Yang Li ${ }^{5}$, \\ Xiaowei Guan ${ }^{6}$, Xiang $\mathrm{Cao}^{1,7}$, Yun X $\mathrm{u}^{1,7}$ and Yonghua Zhao ${ }^{8 *}$
}

1 Department of Neurology, Nanjing Drum Tower Hospital Clinical College of Traditional Chinese and Western Medicine, Nanjing University of Chinese Medicine, Nanjing, China, ${ }^{2}$ Department of Biotherapy, Shenzhen Luohu People's Hospital, Shenzhen, China, ${ }^{3}$ Department of Medical Imaging and Radiological Sciences, Kaohsiung Medical University, Kaohsiung City, Taiwan, ${ }^{4}$ Biomedical Imaging Research Center, National Yang-Ming University, Taipei, Taiwan, ${ }^{5}$ State Key Laboratory of Quality Research in Chinese Medicine, Faculty of Chinese Medicine, Macau University of Science and Technology, Macau, China, ${ }^{6}$ Department of Human Anatomy and Histoembryology, School of Medicine and Life Sciences, Nanjing University of Chinese Medicine, Nanjing, China, ${ }^{7}$ Department of Neurology, Drum Tower Hospital, Medical School and The State Key Laboratory of Pharmaceutical Biotechnology, Institute of Brain Science, Nanjing University, Nanjing, China, ${ }^{8}$ State Key Laboratory of Quality Research in Chinese Medicine, Institute of Chinese Medical Sciences, University of Macau, Macau, China

\section{OPEN ACCESS}

Edited by:

Zhidong Liu,

Tianjin University of Traditional Chinese Medicine, China

Reviewed by:

Haixia Lu,

Xi'an Jiaotong University, China

Kevin Donald Broad, University College London,

United Kingdom

${ }^{*}$ Correspondence: Yonghua Zhao

yonghuazhao@um.edu.mo

Specialty section:

This article was submitted to

Neuropharmacology,

a section of the journal

Frontiers in Neuroscience

Received: 29 January 2019 Accepted: 06 May 2019

Published: 29 May 2019

Citation:

Liu B, Zhang $Q$, Ke C, Xia Z, Luo C, Li Y, Guan X, Cao X, Xu Y and

Zhao $Y$ (2019)

Ginseng-Angelica-Sansheng-Pulvis Boosts Neurogenesis Against Focal

Cerebral Ischemia-Induced Neurological Deficiency.

Front. Neurosci. 13:515.

doi: 10.3389/fnins.2019.00515
Background: The traditional Chinese medicine Ginseng-Angelica-Shanseng-Pulvis (GASP) has been used to treat stroke for 300 years. This present study investigated if it can induce increases in neurogenesis following cerebral ischemic injury.

Methods: Rats following middle cerebral artery occlusion were orally treated with high, medium, and low doses of a standardized GASP extract.

Results: After 14 days, treatment with GASP improved regional blood flow and infarction volume by magnetic resonance imaging scanning, enhanced Ki67+ expression in the subventricular zone, increased brain-derived neurotrophic factor (BDNF) secretion, Nestin, and bone morphogenetic protein (BMP) 2/4 expressions in the hippocampus in a dose-dependent manner. Interestingly, low-dose treatment with GASP downregulated doublecortin and Notch1 expressions in the hippocampus, as well as upregulated glial fibrillary acidic protein expression in the subgranular zone and hairy and enhancer of split (Hes) 5 expression in the hippocampus, while treatment with middle and high doses of GASP reversed these results. Meanwhile, the consumed time was shortened in the basket test and the adhesive removal test and the spending time on exploring novel objects was prolonged by GASP treatment whose effects were more obvious at day 14 post-ischemia.

Conclusion: Our study demonstrates that treatment with GASP increases neurogenesis and ameliorates sensorimotor functions and recognition memory. We hypothesize that these effects are thought be mediated by an effect on the BMP2/4 pathway and Notch1/Hes5 signal. Due to its beneficial efficacy, GASP can be recognized as an alternative therapeutic agent for ischemic stroke.

Keywords: Ginseng-Angelica-Sansheng-Pulvis, ischemic stroke, neurogenesis, neurological deficiency, neural differentiation 


\section{INTRODUCTION}

Cerebrovascular disease seriously threatens human life and health, especially in middle-aged and elderly people. Ischemic stroke has been one of the most common cerebrovascular diseases, with high rates of morbidity and mortality (Kim and Johnston, 2013). The pathology of ischemic stroke is extremely complex, and as a result, there have been few significant advances in the efficacy of treatment options (Roth and Liesz, 2016). Recently, developments have suggested that the regenerative capacity of neural cells following ischemia is highly correlated with functional recovery (Zhang et al., 2001; Thored et al., 2006; Quentin et al., 2015).

Neurogenesis refers to the differentiation of neural stem cells (NSCs) into various types of neural cells, such as neurons and astrocytes. Neurogenesis occurs throughout physiological and pathological progresses. To date, two "reservoirs" of endogenous stem cells have been found in the brain: the subventricular zone (SVZ) of the lateral ventricles and the subgranular zone (SGZ) of the dentate gyrus in the hippocampus, which is important for learning and memory in the brain. Proliferated and differentiated NSCs in the two classical neurogenic niches result in stroke-induced neurogenesis (Quentin et al., 2015). Neurogenesis normally occurs post-stroke, and this is associated with the potential for functional improvement and rehabilitation. In stroke-induced neurogenesis in human ischemic penumbra, newborn neurons take precedence over localizing near blood vessels, where it contributes to establishing a microenvironmental corridor (Jin et al., 2006). Therapeutic outcomes against ischemia are dependent on the period of adult neurogenesis occurring in the SVZ and SGZ. Proliferated cells in the SVZ directly migrate toward the infarction zone and then differentiate and integrate with cerebral parenchyma in adult rodents after stroke (Thored et al., 2006; Yamashita et al., 2006), and then, the stress of middle cerebral artery occlusion (MCAo) stimulates the proliferation of neuronal progenitor cells in the SGZ (Yagita et al., 2001). These studies have demonstrated that maximizing the efficacy of the brain's endogenous mechanisms of self-repair is a promising treatment approach.

Neuroepithelial cells highly express Nestin, which can be recognized as a marker of progenitor cells of both neuronal and glial lineage (Halbach, 2011), while doublecortin (DCX) represents the existence of neuroblasts and immature neurons (Quentin et al., 2015). Reactive astrocyte-like cells marked by glial fibrillary acidic protein (GFAP) are a widespread endogenous stem cellular potential source following brain injury (Robel et al., 2011). A population of $\mathrm{GFAP}^{+} / \mathrm{Nestin}^{+}$radial cells in the SGZ generates self-renewing non-radial type 2 progenitors in turn to give rise to $\mathrm{DCX}^{+}$neuroblast differentiation, indicating a possible reciprocal lineage relationship between DCX and GFAP/Nestinpositive cells (Aimone et al., 2014). In response to the stroke stress, many cytokines such as brain-derived neurotrophic factor (BDNF), vascular endothelial growth factor (VEGF), insulinlike growth factor-1, and fibroblast growth factor-2 participate in proliferation of progenitor cells in both the SVZ and SGZ (Wiltrout et al., 2007). Among them, BDNF is well-known as the key neurotrophic factor not only for neurogenesis but also for assisting in activity-dependent plasticity, i.e., how the various synapses link together after brain injury. It is a potent regulator of neural systems as a whole (Scharfman et al., 2005). Notch1 and bone morphogenetic protein (BMP) signaling pathways are considered as two major regulated approaches of NSCs in neurogenic niches (Jing et al., 2009; Ables et al., 2010). The helixloop-helix gene Hes5 is an essential downstream target gene for Notch signaling, which regulates the maintenance and expansion of NSCs (Ohtsuka et al., 2001), and further downregulation of Hes5 expression induces cellular differentiation in NSC pool (Chiba, 2006).

Ginseng-Angelica-Sansheng-Pulvis (GASP) has been employed as a traditional Chinese treatment for stroke since the early Qing dynasty and is composed of Panax ginseng C.A.Mey., root and rhizome; Angelica sinensis (Oliv). Diels, root, and rhizome; as well as Cinnamomum cassia (L). J.Presl, stem bark. The herbal plants have been used as traditional medicines for 1000s of years in oriental countries and are considered to have the ability of rejuvenating the body. Studies suggest that total saponins derived from ginseng improve neurological deficits after focal cerebral ischemia by the enhancement of neurogenesis (Zheng et al., 2011). Previously, we investigated therapeutic effects of two active constituents of $A$. sinensis: sodium ferulate (SF) and $n$-butylidenephthalate (BP) on stroke in rats, and found that SF could increase the expression of Nestin in the ischemic infarction zone (Zhao et al., 2012). Moreover, combination of SF and BP further increased the expressions of VEGF, BDNF, and neuron-specific class III beta-tubulin in ischemic penumbra and reduced the infarction volume (Zhang et al., 2016). Trans-cinnamaldehyde, an essential component in C. cassia, has a potential neuroprotective effect against ischemic stroke (Chen et al., 2016). Our previous study has demonstrated that GASP extraction is an angiogenic switch, which contributes to reductions of infarction volume and mortality post-stroke (Liu et al., 2018). However, whether GASP extraction can boost neurogenesis after cerebral ischemia is still unknown. In this study, we investigate the efficacy and mechanism of GASP on neurogenesis and hypothesize that BMP2 and 4 and Notch1/Hes5 signal pathways are involved in the procedure of neurogenesis after ischemic stroke.

\section{MATERIALS AND METHODS}

\section{The Quality Control of Ginseng-Angelica-Shanseng-Pulvis}

Panax ginseng C.A.Mey., root and rhizome (bath no. 160101); A. sinensis (Oliv). Diels, root, and rhizome (bath no. 160301); and C. cassia (L). J.Presl, stem bark (bath no. 160101) were obtained from Guangzhou Zisun Pharmaceutical, Co., Ltd., China. The herbal medicines were identified by Professor Quan Zhu and accordant with the standards of Chinese Pharmacopoeia (2015). After the procedure of steam distillation, the aqueous extract was mixed with the volatile oil and consequently was manufactured into GASP extraction. The detailed extracted method of GASP and its representative compounds including ferulic acid, cinnamaldehyde, ligustilide, and ginsenoside Rb1 
TABLE 1 | Major compounds in the volatile oil and (or) Ginseng-AngelicaShanseng-Pulvis (GASP) extraction.

\begin{tabular}{lcc}
\hline Compound & $\boldsymbol{t}_{\mathbf{R}}(\mathbf{m i n})$ & Yield $\mathbf{( m g} \mathbf{g})$ \\
\hline Ferulic acid & 32.46 & $11.7 \pm 0.3$ (GASP) \\
Cinnamaldehyde & 57.34 & $215.2 \pm 0.2$ (volatile oil) $6.5 \pm 0.4$ (GASP) \\
Ligustilide & 81.71 & $172.4 \pm 0.3$ (volatile oil) \\
Ginsenoside Rb1 & 90.15 & $20.0 \pm 0.4$ (GASP) \\
\hline
\end{tabular}

by high-performance liquid chromatography (HPLC) analysis have been reported in our previous article (Liu et al., 2018). Comparison of the retention times with standard compounds (originated from National Institutes for Food and Drug Control, China) and production of main active compounds from volatile oil and (or) GASP extraction are listed in Table 1.

\section{Permanent Middle Cerebral Artery Occlusion Model and Experimental Groups}

Male Sprague-Dawley (SD) rats were purchased from the Laboratory Animal Service Centre of Chinese University of Hong Kong. This study was carried out in accordance with the principles of the NIH Guide for the Care and Use of Laboratory Animals (National Institutes of Health Publication No. 80-23, revised 1996) and recommendations of Animals in Neuroscience Research by the Society for Neuroscience, Institutional Animal Care and Use Committee of Macau University of Science and Technology. The protocol was approved by the Institutional Animal Care and Use Committee of Macau University of Science and Technology. The survey procedure of the MCAo model, enrollment criteria, and laboratory environmental conditions were consistent with previous study (Liu et al., 2018). Briefly, SD rats were anesthetized; subsequently, 4-0 surgical nylon suture coated with polylysine was inserted into the lumen of the right common carotid and advanced into the internal carotid artery until the origin of the middle cerebral artery was occluded, and sham-operated rats underwent the same surgery but without nylon suture inserted.

Forty-eight MCAo rats whose neurological deficit scores were at least two by using a score following the protocol of Menzies et al. (1992) were randomly divided into four groups: the MCAo group, and the low-, middle-, and high-dose GASP groups (12 rats per group). At the same time, six Sham-operated rats were recruited for a Sham group. Three dosages of GASP extraction $(2.3,4.6$, and $9.2 \mathrm{~g} / \mathrm{kg})$ were dissolved into distilled water and prepared for three kinds of suspension as before. Rats in the low, middle, and high dosage groups were subjected to intragastric administration with three kinds of suspension twice a day at 7:00 and 19:00 for 3 days before and 14 days after MCAo. Simultaneously, rats in the MCAo group and Sham group were given the same volume of distilled water.

\section{Behavioral Tests}

All rats were handled and trained five times every day, and it continued 3 days prior to the beginning of subsequent tests. The day before operation, behavioral tests were conducted, and continued at days 4,7 , and 14 after surgery by a blindexperimental operator (Figure 1A).

\section{Basket Test}

It is used to assess motor coordination and sensorimotor deficits in rodent models of central nervous system disorders, and its content originates from http://med.stanford.edu/sbfnl/services/ $\mathrm{bm} / \mathrm{sm} /$ BasketTest.html. Briefly, one rat was placed in the center of the open basket, which was made of horizontal and vertical cross wires with about $2 \mathrm{~cm}^{2}$ gap spacing. Subsequently, the operator inverted the basket. Rats were allowed to climb down the walls of the wire basket into their home cage. The time rats spent on completing the task was recorded, and each test was repeated three times.

\section{Adhesive Removal Test}

The sensitive method by Bouet et al. (2009) is used to evaluate sensorimotor deficits. Briefly, two small pieces of paper tape (of equal size square, $1 \mathrm{~cm}^{2}$ ) were taken as bilateral tactile stimuli to stick to the palmar surface of each forelimb (Figure 1C). The rats were then placed in their cages, and the operator observed the time on which the rats spent removing each tape by their teeth. Before surgery, all rats were able to remove the paper tape within $5 \mathrm{~s}$ at the end of training. The time required from the three repeated trials to remove both stimuli from each limb was recorded, and the mean time was calculated.

\section{Novel Object Recognition}

The novel object recognition test is applied for the evaluation of cognition, particularly recognition memory in rodent models of central nervous system disorders. This test is based on the spontaneous tendency of rodents to spend more time on exploring a novel object rather than a familiar one, which reflects the actions of learning and recognition memory (Huang et al., 2014). The test comprised three parts and was performed for 4 days. The first part was being habituated. On the first and second training days, rats were put into a white box $(40 \mathrm{~cm} \times 40 \mathrm{~cm} \times 30 \mathrm{~cm})$ for $10 \mathrm{~min}$ to habituate to the manipulations and the environment. On the third training day, rats were put into the same box with two identical objects for $10 \mathrm{~min}$ to explore and recognize the objects. At testing day, the final memory test was performed. Rats were individually put into the box with an familiar object and one novel object for $10 \mathrm{~min}$ (Figure 1B). The test was conducted in a quiet environment, and the movements of rats in the box were recorded with a video camera. The ratio of time spent on the novel object was analyzed.

\section{T2W and DSC Examinations by Magnetic Resonance Imaging}

Prior to imaging, rats anesthetized with an initial inhalation of $4 \%$ isoflurane for $3 \mathrm{~min}$ and maintained with $2 \%$ isoflurane in a mixture of $20 \%$ oxygen and $80 \%$ room air were placed in the stereotaxic holder of an MRI machine equipped with a heating system to maintain body temperature and a pressure detector to monitor respiration. At day 14, a Bruker Pharmascan 7-Tesla MR scanner (Bruker Biopsin MRI GmbH, Ettlingen, 
A

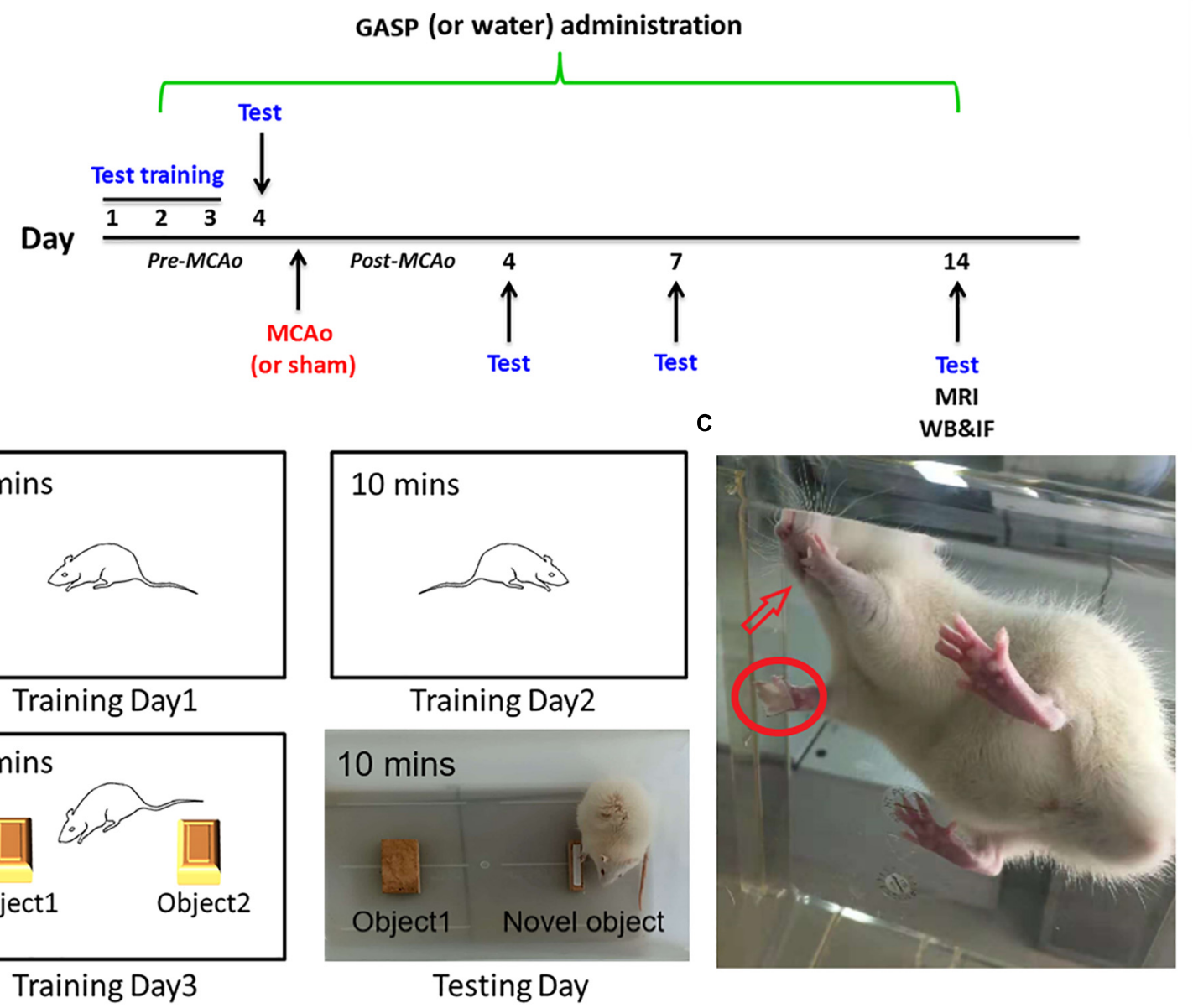

FIGURE 1 | (A) The overall experimental process timeline. (B) The schematic of the novel object recognition test. (C) Rat in adhesive removal testing: the rat finds paper type sticking on its forelimb and tries to remove them using its tooth.

Germany) using a birdcage head coil of $75 \mathrm{~mm}$ inner diameter for radio frequency (RF) transmission and a 20-mm-diameter surface coil for reception was used for MRI scanning. MRI data sets consisting of T2W and DSC were acquired at corresponding time points. A Turbo Rapid Acquisition with Refocused Echoes (RARE) $\mathrm{T}_{2}$-weighted scan was used from Bruker BioSpin MRI $\mathrm{GmbH}$ : repetition time $(\mathrm{TR})=3,000 \mathrm{~ms}$, effective echo time $(\mathrm{TE})=40 \mathrm{~ms}$, rare factor $=4$, number of average $=4$; field of view $(\mathrm{FOV})=20 \mathrm{~mm} \times 20 \mathrm{~mm}$, slice thickness $=1 \mathrm{~mm}$, matrix $=256 \times 256$, scan time $=6.24 \mathrm{~min}$. DSC-MRI data were acquired with GE EPI with $\mathrm{TE}=20 \mathrm{~ms}$, $\mathrm{TR}=1,000 \mathrm{~ms}$, bandwidth $=250 \mathrm{kHz}$, and no averaging. Sixteen contiguous (1 mm thick) slices were recorded in axial orientation with an FOV of $20 \mathrm{~mm} \times 20 \mathrm{~mm}$ and a matrix of $96 \times 96$ to give a nominal resolution of $208 \mu \mathrm{m} \times 208 \mu \mathrm{m}$. A series of 300 images with a temporal resolution of $1,000 \mathrm{~ms}$ were acquired. Eight coronal and eight horizontal slices were acquired covering a volume extending $10 \mathrm{~mm}$ in the rostrocaudal direction in both T2W and DSC. Coronal slices were centered around the infarction lesion, whereas horizontal slices were aligned with the skullcap.

\section{Immunofluorescence Staining}

Rats (Sham group, $n=3$, other groups $n=4$ ) were sacrificed on the 14th day, and their brains were fixed in fresh $4 \%$ PFA. Fresh frozen coronal sections of $8 \mu \mathrm{m}$ thickness were cut by a cryostat microtome (Shandon Cryotome FSE; Thermo Fischer Scientific). A total of six sections were used for immunofluorescence staining in the same position of every rat. To investigate cellular proliferation in the SVZ as well as neurogenesis in the SGZ, SVZ sections were incubated with antibody against Ki67 (Rabbit, 1:1,000; Abcam); three sections of the SGZ were incubated with DCX (Rabbit, 1:2,000; Abcam) overnight at $4^{\circ}$; then, samples were washed with PBS and incubated with the Alexa Fluor ${ }^{\circledR} 488$ goat anti-rabbit immunoglobulin (1:200; Life Technologies) for $1 \mathrm{~h}$. After that, sections were incubated with NeuN (Mouse, 1:3,000; Abcam) in the same condition as DCX and Alexa Fluor ${ }^{\circledR} 594$ goat anti-mouse immunoglobulin (1:200; Life Technologies) secondary antibodies for $1 \mathrm{~h}$ at room temperature. The other three sections of the SGZ with GFAP (Rabbit, 1:2,000; Abcam) and Nestin (Mouse, 1:2,000; Abcam) staining were manipulated in the same way. Nuclei were stained with 4', 6-diamidino-2-phenylindole (DAPI; Sigma). Fluorescent 
labeling was examined with the Leica TCS SP8 laser scanning confocal microscope (Leica Microsystems, Inc., Buffalo Grove, IL, United States). The immunofluorescence positive signals of each slice was evaluated by an investigator who was blind to the experiment under a magnification of $10 \times 20$ in confocal images. Image-Pro Plus software (Media Cybernetics, Rockville, $\mathrm{MD}$, United States) was applied to measure the fluorescent positive density. In control experiments, primary antibodies were replaced with PBS.

\section{Western Blot}

Protein samples were extracted from the hippocampus of each group $(n=3)$ after 14 days of administration. Protein concentration was determined by enhanced bicinchoninic acid (BCA) protein assay kit (Beyotime Institute of Biotechnology, Shanghai, China). Protein samples were electrophoresed on gradient sodium dodecyl sulfate (SDS)-polyacrylamide gel (BioRad) and subsequently electrotransferred to polyvinylidene difluoride (PVDF) membranes (Bio-Rad) in Tris-glycine transfer buffer. Membranes were blocked in 5\% (w/v) non-fat dry milk for $1 \mathrm{~h}$ at room temperature, followed by protein incubation with primary antibodies including anti-DCX, anti-Nestin (Rabbit, 1:1000; Abcam), anti-BDNF, anti-BMP2, anti-BMP4, anti-Hes5 (Rabbit, 1:500; Abcam), and anti-Notch1 (Rabbit, 1:1,000; CST) at $4^{\circ} \mathrm{C}$ overnight. Then, the membranes were gently washed with TBST [50 mM Tris-HCl (pH 7.4; Acros Organics BVBA, Geel, Belgium), $150 \mathrm{mM} \mathrm{NaCl}$, and 0.05\% Tween 20 (Acros Organics BVBA)] three times and incubated with goat anti-rabbit IgG $(\mathrm{H}+\mathrm{L})$ secondary antibodies (1:5,000; LI-COR Biosciences, Lincoln, NE, United States) at room temperature for $1 \mathrm{~h}$. Finally, the membranes were scanned, and the signals of reactive bands were quantified using the Odyssey Infrared Imager (LI-COR). $\beta$-Actin was detected as internal control. Gray value levels of the targeted proteins were quantitatively analyzed by the ratio of corresponding proteins to $\beta$-actin through Image $\mathrm{J}$ software (National Institutes of Health, United States). Western blots were duplicated three independent times.

\section{Statistical Analysis}

All results were expressed as means \pm standard deviation. Two-way repeated measures analysis of variance (ANOVA) was performed to determine the interaction effect between group and time period on related neurological function, and one-way ANOVA was used to analyze other data followed by Tukey test for multiple comparisons. Differences were considered to have statistical significance at $p<0.05$. All data were analyzed using SPSS 19.0 (IBM, Armonk, NY, United States).

\section{RESULTS}

\section{Ginseng-Angelica-Shanseng-Pulvis Extraction Enhanced Sensorimotor Functions and Recognition Memory}

In the basket test, a two-way repeated measures ANOVA showed that significant effects for both group and time period regarding

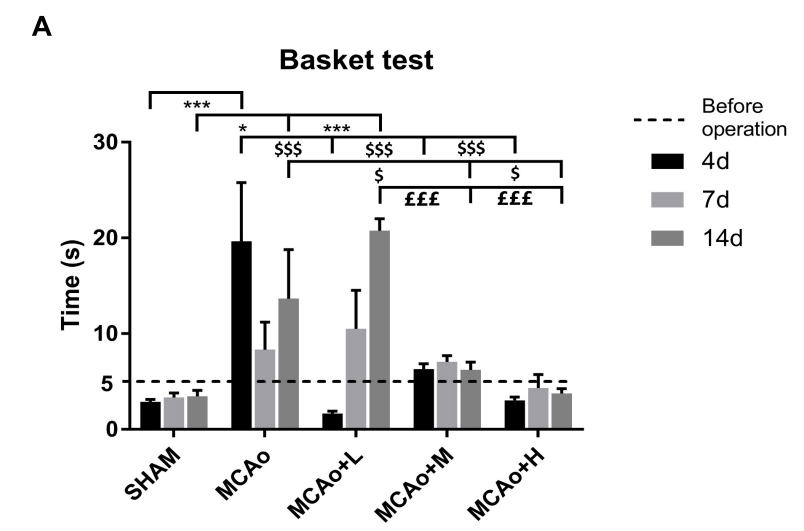

B

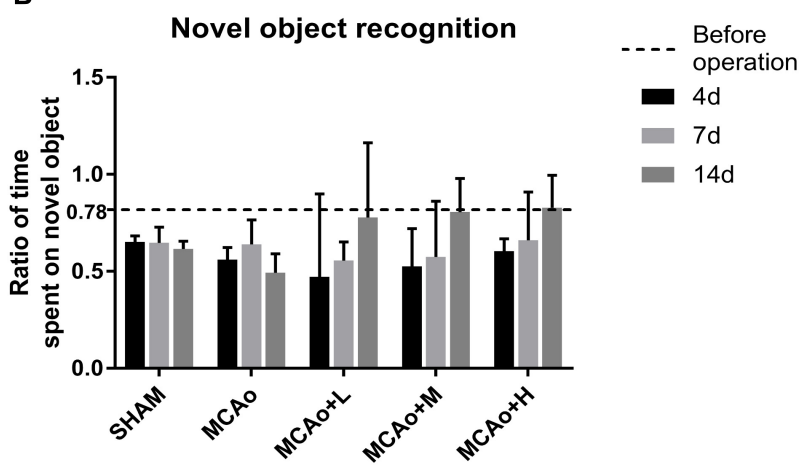

FIGURE 2 | Assessments of basket test and novel object recognition test outcomes. (A) The basket test was performed before operation as well as 4, 7 , and 14 days after operation in each group. (B) Ratio of time spent on novel object was counted at the same time. Data presented as mean \pm standard deviation. ${ }^{*} p<0.05,{ }^{* * *} p<0.001$ vs. Sham group; ${ }^{\$} p<0.05,{ }^{\$} \$ p<0.001$ vs. middle cerebral artery occlusion (MCAo) group; $\mathcal{E \Sigma}_{p}<0.001$ vs. MCAo + Low-dose Ginseng-Angelica-Shanseng-Pulvis (GASP) (L) group.

neurological function of four MCAo operating groups (group: $F=12.12, p<0.001$; time period: $F=2.709, p<0.001)$, as well as obvious effects in the factor of group and time period $(F=5.655, p<0.001)$. As shown in Figure 2A, quantitative analysis indicated that the consumed time of the middle- to highdose group was approximate to that of the Sham group at day 4 , and the trend was always maintained at day 14. During the period from 7 to 14 days, it seemed that the rats in the low-dose group consumed slightly longer than those in the MCAo group, but there was no statistical significance.

The adhesive removal test focused on the sensory function of the forelimbs in rats and the coordination of limb movements. Rats' left forepaw in the MCAo group showed functional deficiency, and the time spent on the right front paw was prolonged to various degrees (Table 2, 4 days: $p<0.05$ vs. SHAM; 14 days: $p<0.01$ vs. SHAM). At day 7 , the right side in the low-dose group resumed, following the left side recovering to a certain degree at day 14 (14 days left: $p<0.05$ vs. SHAM). For rats in the middle- and high-dose groups, left anterior paws showed deficient sensory function only at day 4 , and the function began to be restored at day 7. Moreover, 
TABLE 2 | The result of adhesive removal test.

Time (s)

\begin{tabular}{|c|c|c|c|c|c|c|c|c|}
\hline \multirow[b]{2}{*}{ Groups } & \multicolumn{2}{|c|}{ Before operation } & \multicolumn{2}{|c|}{4 days } & \multicolumn{2}{|c|}{7 days } & \multicolumn{2}{|c|}{14 days } \\
\hline & Left & Right & Left & Right & Left & Right & Left & Right \\
\hline SHAM & $2.3 \pm 0.8$ & $2.6 \pm 1.3$ & $2.8 \pm 1.0$ & $3.0 \pm 0.8$ & $2.5 \pm 1.0$ & $2.5 \pm 0.5$ & $2.2 \pm 0.4$ & $2.2 \pm 0.4$ \\
\hline MCAo & $2.3 \pm 0.8$ & $2.6 \pm 1.3$ & No response & $6.7 \pm 2.3^{*}$ & No response & $3.5 \pm 1.7$ & No response & $6.5 \pm 3.1^{* *}$ \\
\hline $\mathrm{MCAO}+\mathrm{L}$ & $2.3 \pm 0.8$ & $2.6 \pm 1.3$ & No response & No response & No response & $6.0 \pm 6.2$ & $15.0 \pm 15.7^{*}$ & $3.2 \pm 3.4$ \\
\hline $\mathrm{MCAO}+\mathrm{M}$ & $2.3 \pm 0.8$ & $2.6 \pm 1.3$ & No response & $5.1 \pm 5.1$ & $8.7 \pm 6.1^{*}$ & $5.0 \pm 2.9$ & $7.3 \pm 3.3$ & $5.8 \pm 3.9$ \\
\hline $\mathrm{MCAO}+\mathrm{H}$ & $2.3 \pm 0.8$ & $2.6 \pm 1.3$ & No response & $8.8 \pm 7.3$ & $7.5 \pm 4.9$ & $4.7 \pm 3.1$ & $3.5 \pm 1.7^{£}$ & $4.5 \pm 3.9$ \\
\hline
\end{tabular}

Values are mean $\pm S D .{ }^{* *} p<0.01,{ }^{*} p<0.05$ vs. SHAM; ${ }^{£} p<0.05$ vs. MCAo+L. MCAo, middle cerebral artery occlusion.

\section{A CBF deficits (Red zones)}
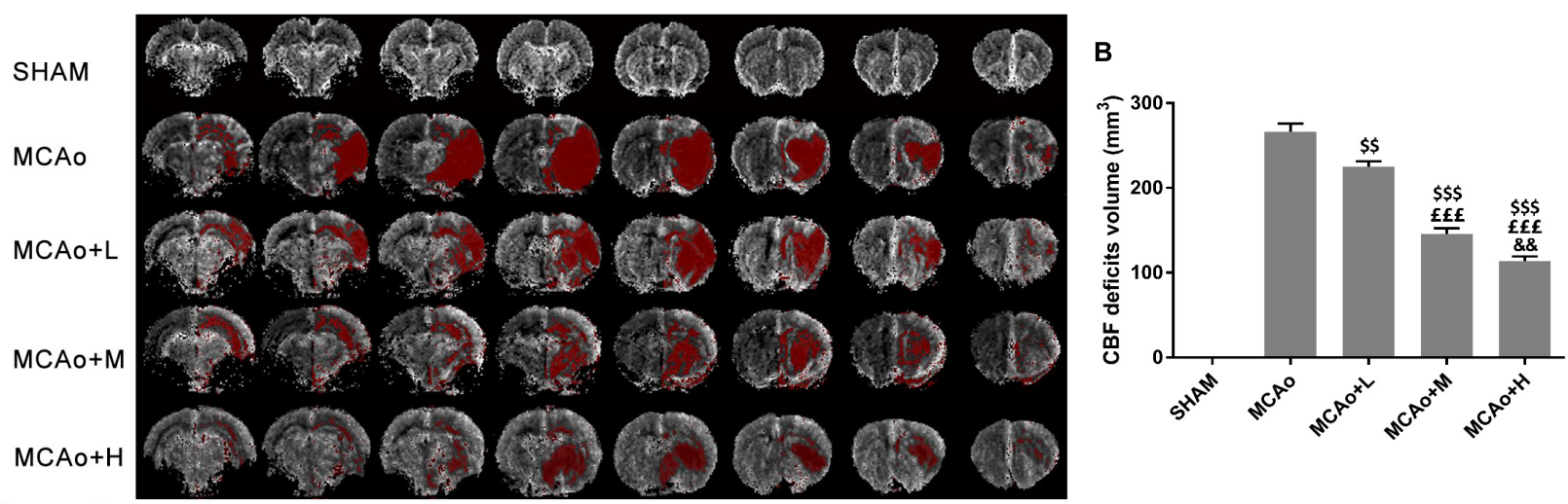

C Infarct volume (Red zones)
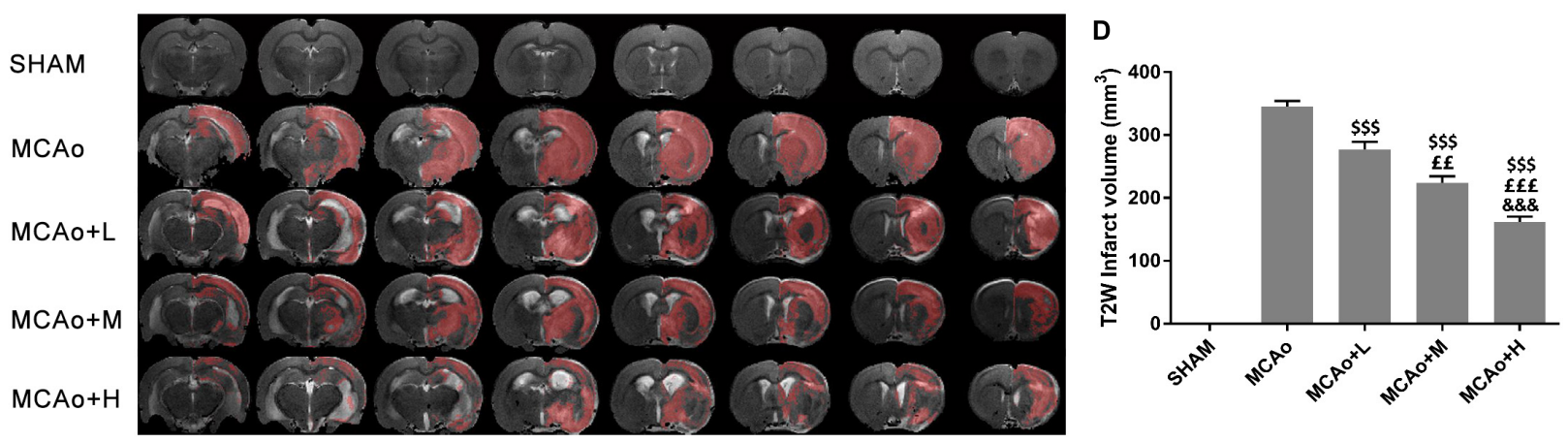

FIGURE 3 | MRI results of regional cerebral blood flow (CBF) deficits and infarction volume evaluation in T2W after 14 days post-MCAo. (A) Regional CBF deficit (red zones) images in each group. (B) Quantitative analysis of CBF deficits. (C) T2W images of infarction volume in different groups. (D) Quantitative analysis of cerebral infarction volume. Data presented as mean \pm standard deviation. $\$ \$ p<0.01,{ }^{\$ \$} p<0.001$ vs. MCAo group; ${ }^{£ £} p<0.01$, ${ }^{£ £ £} p<0.001$ vs. MCAo + Low-dose GASP (L) group; \&\&p < 0.01, \&\&\&p < 0.001 vs. MCAo + Middle-dose GASP (M) group.

the high-dose group's rehabilitated level of sensory function was the best at day 14 .

In the novel object recognition test (Figure 2B), at 14 days, post-stroke learning and memory abilities in the three administration groups further improved. Although the data of each group have no statistical difference, it could reflect the ameliorated trend of recognition memory by GASP extraction treatment.

\section{Ginseng-Angelica-Shanseng-Pulvis Extraction Improved Cerebral Blood Flow and Infarction Volume}

In Figure 3A, DSC images showed a considerable cerebral blood flow (CBF) deficit (red zones) occurring in the ipsilateral hemisphere after stroke. Significantly, the low-, middle-, and high-dose groups ameliorated this status in a dose-dependent 
manner at day 14 (Figure 3B, $p<0.01$, MCAo+L vs. MCAo, $\mathrm{MCAo}+\mathrm{H}$ vs. MCAo+M; $p<0.001, \mathrm{MCAo}+\mathrm{M}$ and $\mathrm{MCAo}+\mathrm{H}$ vs. MCAo and MCAo+L). In T2W images (Figure 3C), the red area in the cerebral cortex and striatum represented infarction volume, whose evolution tendency was consistent with $\mathrm{CBF}$, and the high dose of GASP exerted the most significant advantage on regional $\mathrm{CBF}$ and infarction volume (Figure 3D, $p<0.001$ vs. other groups).

\section{Ginseng-Angelica-Shanseng-Pulvis Extraction Increased Cellular Proliferation in the Subventricular Zone}

$\mathrm{Ki67}$ is recognized as a marker of cellular proliferation. Immunofluorescence staining showed that cells with green immunofluorescence positive signal (indicated by an arrow) distributed in the SVZ of the ipsilateral hemisphere (Figure 4A). Quantitative analysis indicated that GASP enhanced the expression of Ki67 in a dose-dependent manner; obviously, Ki67 expressions in the middle- and high-dose groups were higher than those in the low-dose and MCAo groups (Figure 4B, $p<0.01)$. The results illustrated that GASP extraction can promote cellular proliferative activity in the SVZ.

\section{Ginseng-Angelica-Shanseng-Pulvis Extraction Enhanced the Expressions of Doublecortin, Glial Fibrillary Acidic Protein, and Nestin and Brain-Derived Neurotrophic Factor Secretion}

As shown in Figure $\mathbf{5 A}$, cells with green DCX immunofluorescence positive signal distributed in the SGZ.
Quantitative analysis of both immunofluorescence staining (Figure 5B) and Western blotting (Figures 6A,C) indicated that the expression of DCX in the middle- and high-dose groups was significantly higher than that of the low-dose and MCAo groups. Interestingly, immunofluorescence staining suggested that the low-dose group decreased DCX expression compared with the MCAo group $(p<0.001)$, and the trend of DCX expression was also presented by Western blot assay. However, the doublestaining result indicated that DCX expression in NeuN-positive cells in the low-dose group was obviously higher than that in the MCAo group (Figure 5C, $p<0.001$ ), suggesting that the low dose of GASP contributed to promoting neuronal immaturity. Additionally, Western blot results showed that stress of stroke also stimulated more BDNF secretion in the hippocampus of the ipsilateral hemisphere, and the increased trend of BDNF secretion was dose-dependent after administration of GASP at day 14 (Figures 6A,D; $p<0.001$ ).

As shown in Figure 5D, cells with green $\mathrm{GFAP}^{+}$ immunofluorescence signal are located in the SGZ. Quantitative analysis showed that the expression of GFAP in the lowdose group was higher than that in the middle-dose group $(p<0.05)$, and although GFAP expression in the high-dose group was the highest $(p<0.001$ vs. SHAM, MCAo; $p<0.01$ vs. $\mathrm{MCAo}+\mathrm{M})$, there was no statistical significance between the low- and the high-dose groups (Figure 5E). Qualitative analysis of Nestin expression in three administration groups showed an increasing trend in a dose-dependent manner whether in immunofluorescence staining (Figure 5F) or in Western blot (Figure 6B). These might be explained by the fact that the efficacies of different dosage of GASP on NSCs were distinct: a low dose perhaps contributes to NSC differentiation into

A

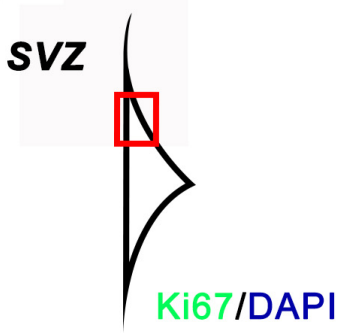

MCAO+L

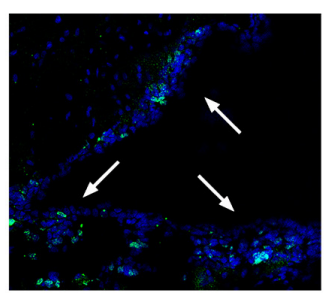

SHAM

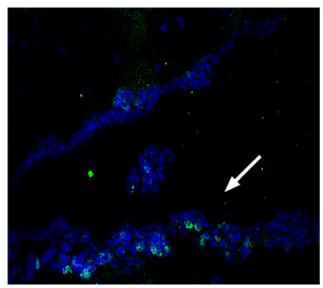

$\mathrm{MCAO}+\mathrm{M}$

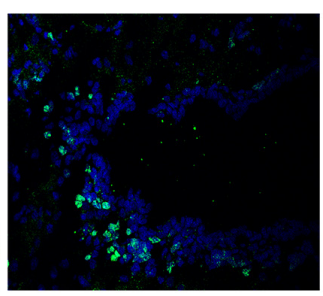

MCAO

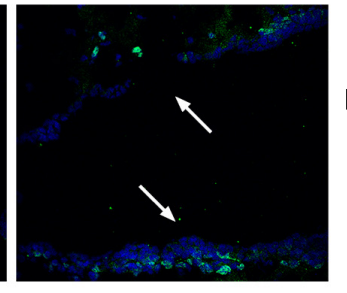

$\mathrm{MCAO}+\mathrm{H}$

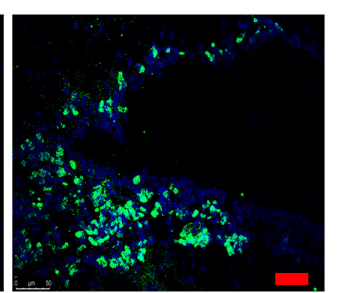

\section{B}

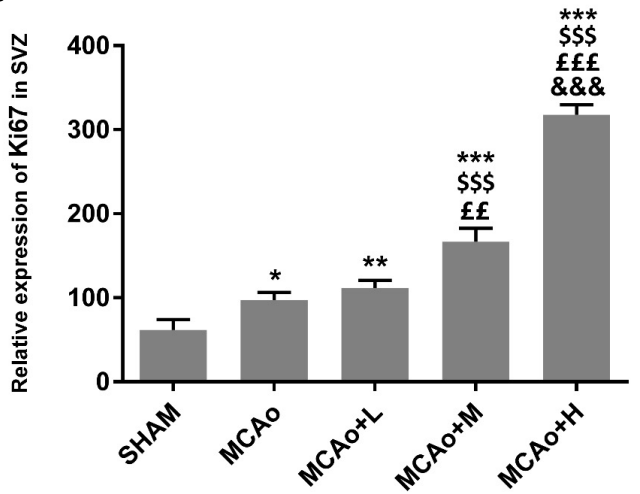

FIGURE 4 | Ginseng-Angelica-Shanseng-Pulvis extraction advanced cell proliferation in the subventricular zone (SVZ) of the ipsilateral hemisphere.

(A) Immunofluorescence staining with Ki67 (green) was presented (magnification $\times 200$ ). (B) Relative expression of Ki67 in the SVZ was analyzed. Scale bar: $50 \mu \mathrm{m}$. Data presented as mean \pm standard deviation. ${ }^{*} p<0.05$, ${ }^{* *} p<0.01,{ }^{* * *} p<0.001$ vs. Sham group; $\$ \$ \$ p<0.001$ vs. MCAo group; ${ }^{\circledR \Sigma} p<0.01, £ £ £ p<0.001$ vs. MCAo + Low-dose GASP (L) group; ${ }^{\& \&} p<0.001$ vs. MCAo + Middle-dose GASP (M) group. 


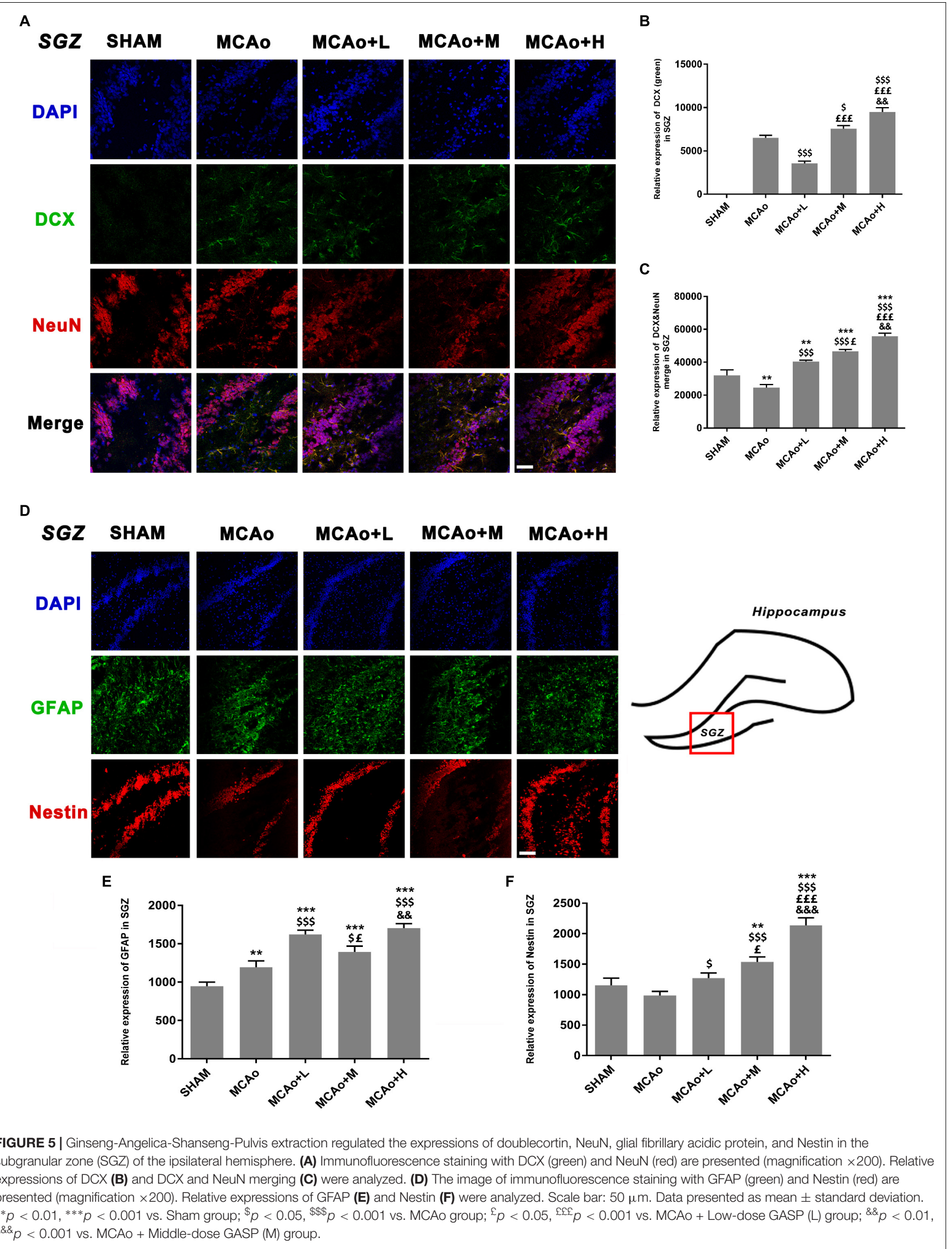


A

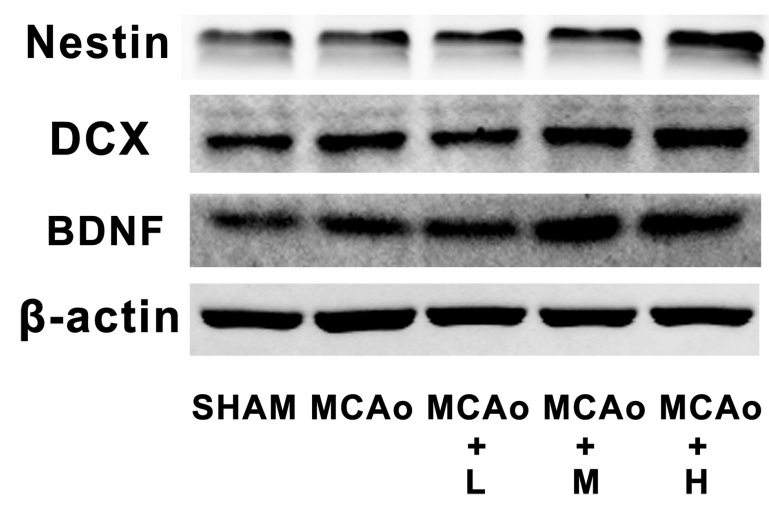

C

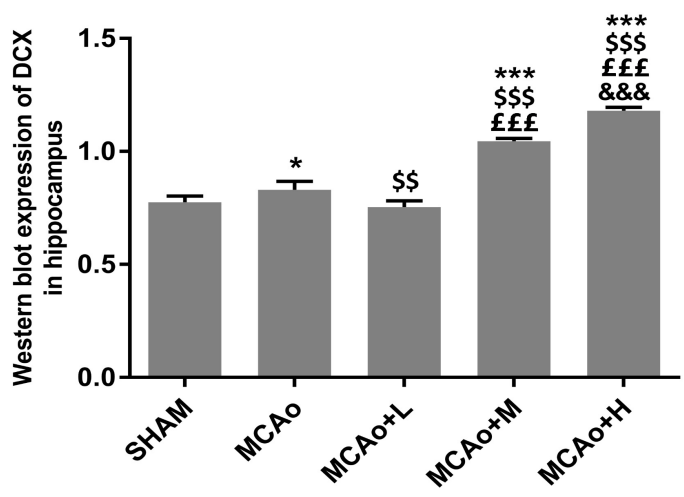

B

$200 k D a$

$45 \mathrm{kDa}$

$15 k D a$

$44 \mathrm{kDa}$

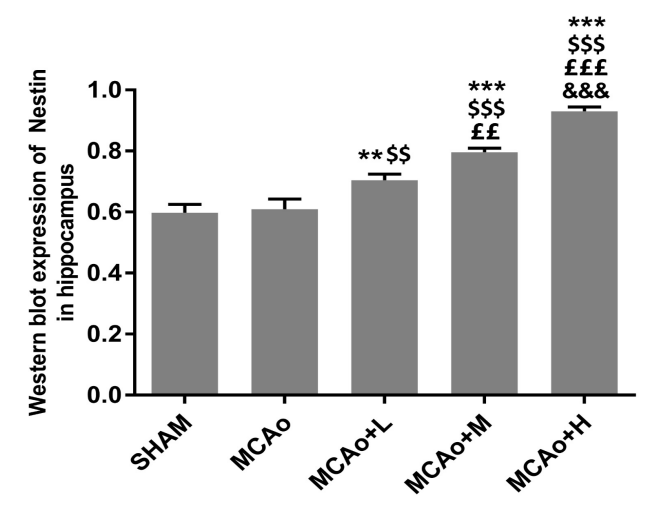

D

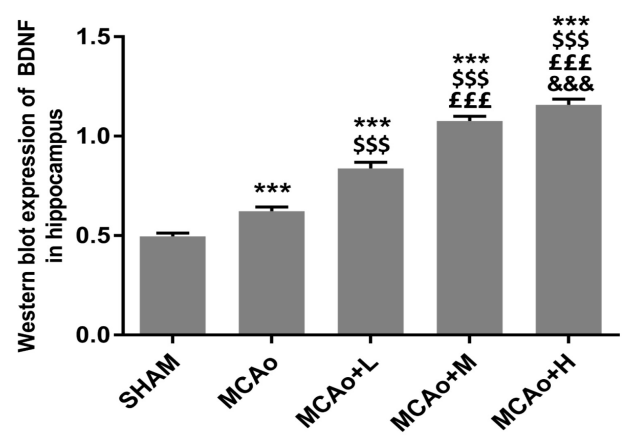

FIGURE 6 | Ginseng-Angelica-Shanseng-Pulvis extraction regulated hippocampal Nestin, DCX, and brain-derived neurotrophic factor (BDNF) expressions. (A) Representative Western blot results for Nestin, DCX, and BDNF in each group's hippocampus and quantitative analysis for Nestin (B), DCX (C), and BDNF (D).

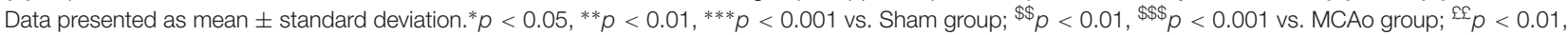
E££ $p<0.001$ vs. MCAo + Low-dose GASP (L) group; \&\&\& $<0.001$ vs. MCAo + Middle-dose GASP (M) group.

glia cells in the SGZ, while a high dose not only enhanced NSCs along with radial glia cellular differentiation, but also advanced NSCs to differentiate into non-radial type 2 cells. Interestingly, the action of middle dose on NSC differentiation appeared in the transformed process from radial glia cells to non-radial type 2 cells.

\section{Ginseng-Angelica-Shanseng-Pulvis Extraction Regulated Bone Morphogenetic Protein 2/4 and Notch1/Hairy and Enhancer of Split 5 Signal Pathways in the Hippocampus}

In order to clarify mechanisms of GASP extraction on neurogenesis, we investigated key signal regulation pathways. As shown in Figures 7A,B, GASP enhanced the expressions of BMP2 and BMP4 in a dose-dependent manner compared with the MCAo group $(p<0.001)$. Surprisingly, the lowdose group inhibited Notch1 and increased Hes5 expressions (Figures 7A,C,D; $p<0.001$ ), together with its high expression of GFAP in the SGZ (Figures 5D,E), indicating that astrocyte-like differentiation was related to suppressed Notch1 and enhanced
Hes5 signals, which was consistent with Guo et al. (2009) results. In contrast, the trend was reversed in the middle- and high-dose groups. The high-dose group promoted Notch1 expression and suppressed Hes5 expression more significantly compared with the middle-dose group $(p<0.01)$.

\section{DISCUSSION}

These data demonstrate that GASP increases neurogenesis by increasing the proliferative activity of cells in the SVZ and the potential of cells to differentiate in the post-ischemic SGZ. This is associated with significant improvements to regional $\mathrm{CBF}$, decreases in cerebral infarction volume, and an enhancement of BDNF secretion in the hippocampus. Improvements to sensorimotor and recognition memory were also observed. These may be related to the activation of BMP2 and BMP4 and regulation of Notch1/Hes5 pathways in the hippocampal zone of the ipsilateral hemisphere (Figure 8).

Accumulating evidence suggests that stress of stroke can stimulate cellular proliferation in the SVZ and neurogenesis (Zhang et al., 2007; Parent et al., 2010), and SVZ cellular proliferative markers gradually increase and peak at day 14 in 


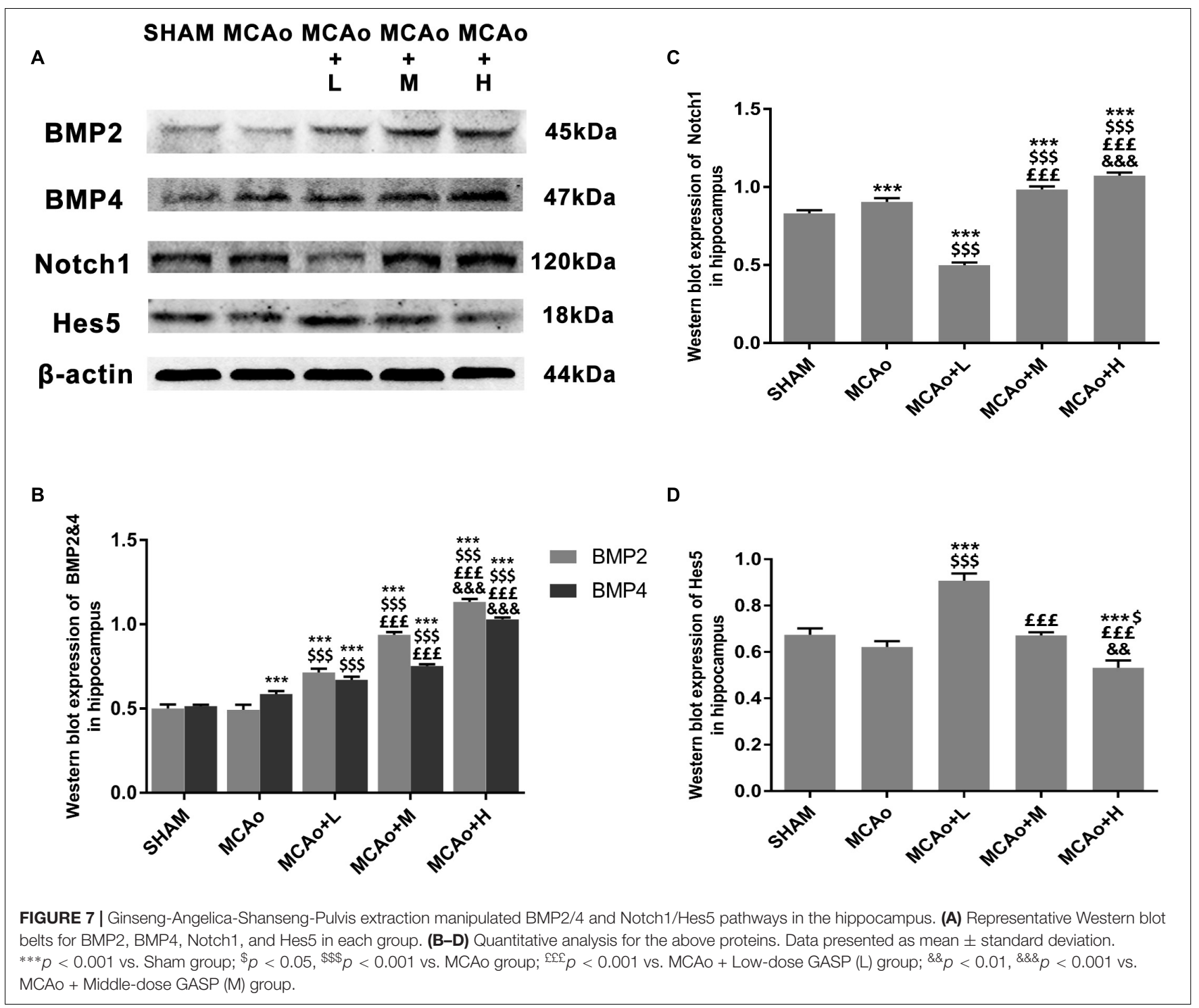

rats with stroke (Zhang et al., 2001). Newly matured neurons found in the ischemic striatum and cortex may be related to their neuronal migration from the SVZ (Thored et al., 2006; Yamashita et al., 2006). We used Ki67, an endogenous proliferative marker, to assess SVZ cellular proliferative ability at day 14 post-MCAo. The result of immunofluorescence staining showed that Ki67 expression in the SVZ began to increase after stroke, which indicated that cellular proliferative ability was activated, and this efficacy was further amplified by the administration of GASP in a dose-dependent manner.

Nestin has been widely accepted as a primary marker of progenitor/stem cell populations (Halbach, 2011). Our Western blot and immunofluorescence staining results showed that the administration of GASP advanced Nestin expression to grow steadily with the dose-dependent trend, suggesting that GASP could obviously increase the number of progenitor/stem cell populations in the hippocampus. The progenitor/stem cells usually differentiate into radial glia cells, which express the astrocytic marker, e.g., GFAP, and/or non-radial type 2 precursor cells, which express DCX (a classic immature neuronal marker) (Mu et al., 2010). In the present study, both Western blot and immunofluorescence staining results showed that the expressions of DCX and DCX merged with NeuN (a mature neuron marker) in the middle- and high-dose GASP groups were significantly higher, showing that the dosage scope boosted NSCs/progenitor cells to differentiate into immature neurons and promoted their maturity. Interestingly, the low-dose group decreased DCX expression, but its expression of DCX-merged NeuN was higher than that of the MCAo group, indicating that the treatment group did not facilitate the precursor cell lineage to differentiate into immature neurons and only slightly elevate maturity of $\mathrm{DCX}^{+}$cells. Moreover, we are surprised to find that in the SGZ, GFAP immunofluorescence expression in the low-dose group was higher than that in the middle-dose group and approximate to the high-dose group, suggesting that low-dose GASP increased the NSCs' differentiated ability along with radial glia cell lineage. 


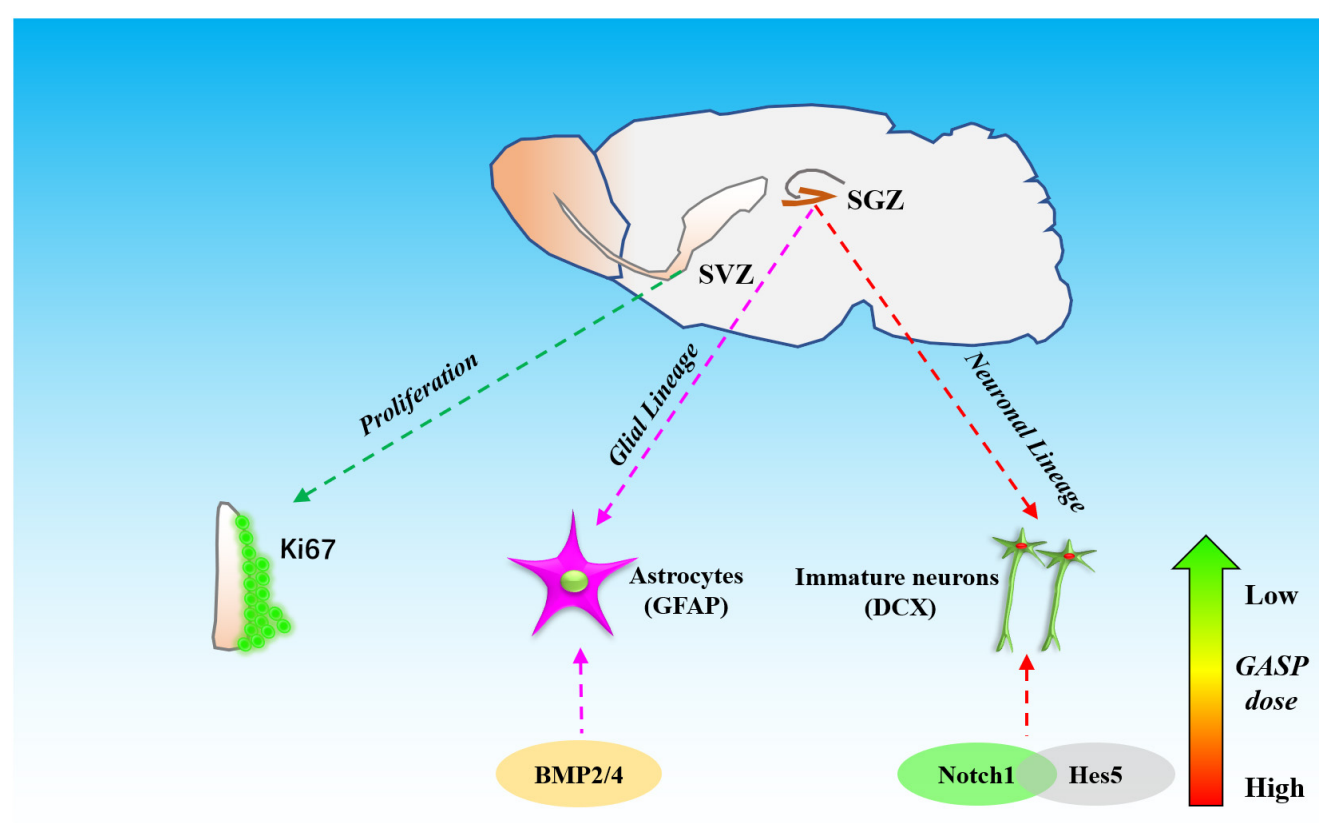

FIGURE 8 | The schematic diagram of different effects of three dosages of GASP on neurogenesis in SGZ and SVZ after ischemic stroke.

Increasing stroke-induced neurogenesis contributes to the improvement of neurological functional outcomes (Leker et al., 2007). Ginsenoside Rg1 enhances ischemia-induced cell proliferation in the dentate gyrus and improves neurological function (Gao et al., 2010). Our previous study also found that combination treatment of SF and BP derived from A. sinensis notably advanced neurogenesis in ischemic boundary zones and ameliorated neurological function and infarction volume (Zhang et al., 2016). Meanwhile, Transcinnamaldehyde, an essential oil in cinnamon powder, also exerts neuroprotective effects and reduces infarction against ischemic stroke (Chen et al., 2016). Our herbal extraction contains these active ingredients, and we have demonstrated that its use improved sensorimotor and object recognition at both high and middle concentrations. We hypothesize that these effects are associated with the promotion of neuron-like differentiation in the hippocampus. Although low-dose GASP enhances astrocyte-like differentiation, it appears to have a negative effect on neurological functional amelioration after stroke.

Additionally, BDNF exerts important actions in protecting brain tissue against injury as well as facilitating neuronal plasticity and neurogenesis. In the hippocampus, it plays a crucial role in learning and memory (Yamada and Nabeshima, 2003). A previous study discovered that exogenous administration of BDNF by intrahippocampal infusion could increase neurogenesis in adult rats (Scharfman et al., 2005). Therefore, BDNF plays an essential role in promoting neurogenesis, and our study shows that its effects are mediated in a dose-dependent manner. Combining the ameliorated sensorimotor and recognition memory in the present study, BDNF further aims to improve behavioral assessment by GASP.

Notch1 and BMP2/4 are two important genes regulating NSC/progenitor cell differentiation signal pathways. Overexpression of Notch1 promotes proliferation of neural progenitor cells (Breunig et al., 2007). It suggests that elimination of Notch1 from inducible Nestin ${ }^{+}$mice decreases NSC number and neuro-like differentiation in the SGZ (Ables et al., 2010). Through the silencing of Hes5, a repressor for tissue-specific gene transcription and the target gene of Notch, proneurogenic Bcl6 recruits SIRT1 to advance neurogenesis (Tiberi et al., 2012). Further research reports that in Hes1-, Hes3-, and Hes5-deficient embryos, neuroepithelial cells are not properly maintained, and radial glial cells are accelerated to differentiate into neurons (Hatakeyama et al., 2004). It suggests that suppression of Hes-5 contributes to neuro-like differentiation. Presently, we demonstrated that significant upregulation of Notchl and downregulation of Hes5 in the high-dose GASP group resulted in the promotion of NSC/progenitor cell division into neuron-like cells. In turn, low-dose GASP decreased the level of Notch1 but reversed its target Hes5 in the hippocampus. Guo and colleagues found that chronic unpredicted mild stress causes decreasing number of neurogenically differentiating cells in the SGZ of adult ischemic rats after 28 days, but it accelerated astrocyte-like cell differentiation by suppressing Notch 1 and enhancing Hes1 and Hes5 expressions (Guo et al., 2009). Therefore, we think that numerous $\mathrm{GFAP}^{+}$cells of the SGZ in the low-dose group may be attributed to inhibited Notch1 and activated Hes5 signals.

The BMP signaling pathway also plays a key regulatory role in different stages of central nervous system development. During 
embryonic neural development, knockdown of BMP2/4/7 in Xenopus embryos caused ubiquitous neural induction throughout the ectoderm (Reversade and Robertis, 2005), and different BMP receptors had varying roles in controlling neural precursor cell production and fate (Panchision et al., 2001). BMP2 and BMP4 can promote adult neural precursor cells differentiating into astrocyte (Gross et al., 1996). Our results indicated that GASP upregulated both BMP2 and BMP4 in a dose-dependent manner in the SGZ, which can be used to explain why high dose induced obvious astrocytelike cell differentiation. Although BMP2/4 expression in the low-dose group was lower than those in the middle- and high-dose groups, combined with downregulation of Notch1 signal, it boosted NSC/progenitor cell differentiation along with astrocytic direction. Therefore, different dosages of GASP determine cell division fate in the SGZ after ischemic stroke. In the present study, there are some limitations that need to be elucidated in future studies. For example, we did not test the differentiated abilities and mechanisms of neuroblasts derived from the SVZ, so we will focus on research following experiments.

In summary, traditionally, we demonstrate a GASP extract in advancing neurogenesis. BMP2/4 and Notch1/Hes 5 pathways are involved in its regulation mechanisms. Due to its beneficial efficacy, GASP shows considerable promise as an alternative therapeutic agent for ischemic stroke.

\section{REFERENCES}

Ables, J. L., Decarolis, N. A., Johnson, M. A., Rivera, P. D., Gao, Z., Cooper, D. C., et al. (2010). Notchl is required for maintenance of the reservoir of adult hippocampal stem cells. J. Neurosci. 30, 10484-10492. doi: 10.1523/ JNEUROSCI.4721-09.2010

Aimone, J. B., Li, Y., Lee, S. W., Clemenson, G. D., Deng, W., and Gage, F. H. (2014). Regulation and function of adult neurogenesis: from genes to cognition. Physiol. Rev. 94, 991-1026. doi: 10.1152/physrev.00004.2014

Bouet, V., Boulouard, M., Toutain, J., Divoux, D., Bernaudin, M., SchumannBard, P., et al. (2009). The adhesive removal test: a sensitive method to assess sensorimotor deficits in mice. Nat. Protoc. 4:1560. doi: 10.1038/nprot. 2009.125

Breunig, J. J., Silbereis, J., Vaccarino, F. M., Šestan, N., and Rakic, P. (2007). Notch regulates cell fate and dendrite morphology of newborn neurons in the postnatal dentate gyrus. Proc. Natl. Acad. Sci. U.S.A. 104, 20558-20563. doi: $10.1073 /$ pnas.0710156104

Chen, Y. F., Wang, Y. W., Huang, W. S., Lee, M. M., Wood, W. G., Leung, Y. M., et al. (2016). Trans-cinnamaldehyde, an essential oil in cinnamon powder, ameliorates cerebral ischemia-induced brain injury via inhibition of neuroinflammation through attenuation of inos, cox-2 expression and nfk-b signaling pathway. Neuromolecular Med. 18, 322-333. doi: 10.1007/s12017016-8395-9

Chiba, M. D. (2006). Concise review: notch signaling in stem cell systems. Stem Cells 24, 2437-2447. doi: 10.1634/stemcells.2005-0661

Gao, X.-Q., Yang, C.-X., Chen, G.-J., Wang, G.-Y., Chen, B., Tan, S.-K., et al. (2010). Ginsenoside Rb1 regulates the expressions of brain-derived neurotrophic factor and caspase- 3 and induces neurogenesis in rats with experimental cerebral ischemia. J. Ethnopharmacol. 132, 393-399. doi: 10.1016/j.jep.2010.07.033

Gross, R. E., Mehler, M. F., Mabie, P. C., Zang, Z., Santschi, L., and Kessler, J. A. (1996). Bone morphogenetic proteins promote astroglial lineage commitment by mammalian subventricular zone progenitor cells. Neuron 17, 595-606. doi: 10.1016/s0896-6273(00)80193-2

Guo, Y. J., Zhang, Z. J., Wang, S. H., Sui, Y. X., and Sun, Y. (2009). Notch1 signaling, hippocampal neurogenesis and behavioral responses to chronic unpredicted

\section{ETHICS STATEMENT}

This study was carried out in accordance with the principles of the NIH Guide for the Care and Use of Laboratory Animals (National Institutes of Health Publication No. 80-23, revised 1996) and recommendations of Animals in Neuroscience Research by the Society for Neuroscience, Institutional Animal Care and Use Committee of Macau University of Science and Technology. The protocol was approved by the Institutional Animal Care and Use Committee of Macau University of Science and Technology.

\section{AUTHOR CONTRIBUTIONS}

YZ designed the research. BL, ZX, CL, and YL completed by grouping, modeling, and administration of experimental animals. QZ and CK finished MRI detection and analysis. BL wrote the manuscript. Thanks to YZ, XG, XC, and YX for their valuable suggestions in the revision and improvement of the manuscript.

\section{FUNDING}

This work was supported by the Science and Technology Development Fund of Macau (FDCT: Grant No. 106/2014/A3).

mild stress in adult ischemic rats. Prog. Neuropsychopharmacol. Biol. Psychiatry 33, 688-694. doi: 10.1016/j.pnpbp.2009.03.022

Halbach, O. V. B. U. (2011). Immunohistological markers for proliferative events, gliogenesis, and neurogenesis within the adult hippocampus. Cell Tissue Res. 345, 1-19. doi: 10.1007/s00441-011-1196-4

Hatakeyama, J., Bessho, Y., Katoh, K., Ookawara, S., Fujioka, M., Guillemot, F., et al. (2004). Hes genes regulate size, shape and histogenesis of the nervous system by control of the timing of neural stem cell differentiation. Development 131, 5539-5550. doi: 10.1242/dev.01436

Huang, T. N., Chuang, H. C., Chou, W. H., Chen, C. Y., Wang, H. F., Chou, S. J., et al. (2014). Tbr1 haploinsufficiency impairs amygdalar axonal projections and results in cognitive abnormality. Nat. Neurosci. 17, 240-247. doi: 10.1038/nn. 3626

Jin, K., Wang, X., Xie, L., Mao, X. O., Zhu, W., Wang, Y., et al. (2006). Evidence for stroke-induced neurogenesis in the human brain. Proc. Natl. Acad. Sci. U.S.A. 103, 13198-13202. doi: 10.1073/pnas.0603512103

Jing, N. H., Sheng, N. Y., and Xie, Z. H. (2009). The function of bmp signaling pathway during the central nervous system development. Chin. J. Cell Biol. 74, 85-97.

Kim, A. S., and Johnston, S. C. (2013). Temporal and geographic trends in the global stroke epidemic. Stroke 44(6 Suppl. 1), S123-S125.

Leker, R. R., Soldner, F., Velasco, I., Gavin, D. K., Androutsellis-Theotokis, A., and McKay, R. D. (2007). Long-lasting regeneration after ischemia in the cerebral cortex. Stroke 38, 153-161. doi: 10.1161/01.str.0000252156.65953.a9

Liu, B., Luo, C., Zheng, Z., Xia, Z., Zhang, Q., Ke, C., et al. (2018). Shengui sansheng san extraction is an angiogenic switch via regulations of AKT/mTOR, ERK1/2 and Notch1 signal pathways after ischemic stroke. Phytomedicine 44, 20-31. doi: 10.1016/j.phymed.2018.04.025

Menzies, S. A., Hoff, J. T., and Betz, A. L. (1992). Middle cerebral artery occlusion in rats: a neurological and pathological evaluation of a reproducible model. Neurosurgery 31, 106-107.

$\mathrm{Mu}$, Y., Lee, S. W., and Gage, F. H. (2010). Signaling in adult neurogenesis. Curr. Opin. Neurobiol. 20, 416-423. doi: 10.1016/j.conb.2010.04.010

Ohtsuka, T., Sakamoto, M., Guillemot, F., and Kageyama, R. (2001). Roles of the basic helix-loop-helix genes Hes1 and Hes5 in expansion of neural stem cells 
of the developing brain. J. Biol. Chem. 276, 30467-30474. doi: 10.1074/jbc. m102420200

Panchision, D. M., Pickel, J. M., Studer, L., Lee, S. H., and Mckay, R. D. (2001). Sequential actions of bmp receptors control neural precursor cell production and fate. Genes Dev. 15, 2094-2110. doi: 10.1101/gad.894701

Parent, J. M., Vexler, Z. S., Gong, C., Derugin, N., and Ferriero, D. M. (2010). Rat forebrain neurogenesis and striatal neuron replacement after focal stroke. Ann. Neurol. 52, 802-813. doi: 10.1002/ana.10393

Quentin, M., Sebastien, V., Renaud, V., and Brigitte, M. (2015). Mechanisms and functional significance of stroke-induced neurogenesis. Front. Neurosci. 9:458. doi: 10.3389/fnins.2015.00458

Reversade, B., and Robertis, E. M. D. (2005). Regulation of admp and bmp2/4/7 at opposite embryonic poles generates a self-regulating morphogenetic field. Cell 123, 1147-1160. doi: 10.1016/j.cell.2005.08.047

Robel, S., Berninger, B., and Götz, M. (2011). The stem cell potential of glia: lessons from reactive gliosis. Nat. Rev. Neurosci. 12, 88-104. doi: 10.1038/nrn2978

Roth, S., and Liesz, A. (2016). Stroke research at the crossroads-where are we heading? Swiss Med. Wkly. 146:w14329. doi: 10.4414/smw.2016.14329

Scharfman, H., Goodman, J., Macleod, A., Phani, S., Antonelli, C., and Croll, S. (2005). Increased neurogenesis and the ectopic granule cells after intrahippocampal BDNF infusion in adult rats. Exp. Neurol. 192, 348-356. doi: 10.1016/j.expneurol.2004.11.016

Thored, P., Arvidsson, A., Cacci, E., Ahlenius, H., Kallur, T., Darsalia, V., et al. (2006). Persistent production of neurons from adult brain stem cells during recovery after stroke. Stem Cells 24, 739-747. doi: 10.1634/stemcells.2005-0281

Tiberi, L., van den Ameele, J., Dimidschstein, J., Piccirilli, J., Gall, D., Herpoel, A., et al. (2012). BCL6 controls neurogenesis through Sirt1-dependent epigenetic repression of selective notch targets. Nat. Neurosci. 15, 1627-1635. doi: 10.1038/ nn. 3264

Wiltrout, C., Lang, B., Yan, Y., Dempsey, R. J., and Vemuganti, R. (2007). Repairing brain after stroke: a review on post-ischemic neurogenesis. Neurochem. Int. 50, 1028-1041. doi: 10.1016/j.neuint.2007.04.011

Yagita, Y., Kitagawa, K., Ohtsuki, T., Takasawa, K., Miyata, T., Okano, H., et al. (2001). Neurogenesis by progenitor cells in the ischemic adult rat hippocampus. Stroke 32:1890. doi: 10.1161/01.str.32.8.1890

Yamada, K., and Nabeshima, T. (2003). Brain-derived neurotrophic factor/TrkB signaling in memory processes. J. Pharmacol. Sci. 91:267. doi: 10.1254/ jphs.91.267
Yamashita, T., Ninomiya, M., Hernandez Acosta, P., Garcia-Verdugo, J. M., Sunabori, T., Sakaguchi, M., et al. (2006). Subventricular zone-derived neuroblasts migrate and differentiate into mature neurons in the post-stroke adult striatum. J. Neurosci. 26, 6627-6636. doi: 10.1523/jneurosci.0149-06. 2006

Zhang, Q., Zhao, Y., Xu, Y., Chen, Z., Liu, N., Ke, C., et al. (2016). Sodium ferulate and n-butylidenephthalate combined with bone marrow stromal cells (BMSCs) improve the therapeutic effects of angiogenesis and neurogenesis after rat focal cerebral ischemia. J. Transl. Med. 14:223. doi: 10.1186/s12967-016-0979-5

Zhang, R., Zhang, Z. G., Zhang, L., and Chopp, M. (2001). Proliferation and differentiation of progenitor cells in the cortex and the subventricular zone in the adult rat after focal cerebral ischemia. Neuroscience 105, 33-41. doi: 10.1016/s0306-4522(01)00117-8

Zhang, R. L., LeTourneau, Y., Gregg, S. R., Wang, Y., Toh, Y., Robin, A. M., et al. (2007). Neuroblast division during migration toward the ischemic striatum: a study of dynamic migratory and proliferative characteristics of neuroblasts from the subventricular zone. J. Neurosci. 27:3157. doi: 10.1523/jneurosci.496906.2007

Zhao, Y., Guan, Y., Xu, Y., Li, Y., and Wu, W. (2012). Sodium ferulate combined with bone marrow stromal cell treatment ameliorating rat brain ischemic injury after stroke. Brain Res. 1450, 157-165. doi: 10.1016/j.brainres.2012. 02.053

Zheng, G. Q., Cheng, W., Wang, Y., Wang, X. M., Zhao, S. Z., Zhou, Y., et al. (2011). Ginseng total saponins enhance neurogenesis after focal cerebral ischemia. J. Ethnopharmacol. 133, 724-728. doi: 10.1016/j.jep.2010. 01.064

Conflict of Interest Statement: The authors declare that the research was conducted in the absence of any commercial or financial relationships that could be construed as a potential conflict of interest.

Copyright (c) $2019 \mathrm{Liu}$, Zhang, Ke, Xia, Luo, Li, Guan, Cao, Xu and Zhao. This is an open-access article distributed under the terms of the Creative Commons Attribution License (CC BY). The use, distribution or reproduction in other forums is permitted, provided the original author(s) and the copyright owner(s) are credited and that the original publication in this journal is cited, in accordance with accepted academic practice. No use, distribution or reproduction is permitted which does not comply with these terms. 